\title{
Deformation coproducts and differential maps
}

by

\author{
R. L. Hudson (Loughborough) and S. Pulmannová (Bratislava)
}

\begin{abstract}
Let $\mathcal{T}$ be the Itô Hopf algebra over an associative algebra $\mathcal{L}$ into which the universal enveloping algebra $\mathcal{U}$ of the commutator Lie algebra $\mathcal{L}$ is embedded as the subalgebra of symmetric tensors. We show that there is a one-to-one correspondence between deformations $\Delta[h]$ of the coproduct in $\mathcal{T}$ and pairs $(\vec{d}[h], \overleftarrow{d}[h])$ of right and left differential maps which are deformations of the differential maps for $\mathcal{T}$ [Hudson and Pulmannová, J. Math. Phys. 45 (2004)]. Corresponding to the multiplicativity and coassociativity of $\Delta[h], \vec{d}[h]$ and $\overleftarrow{d}[h]$ satisfy the Leibniz-Itô formula and a mutual commutativity condition. $\Delta[h]$ is recovered from $\vec{d}[h]$ and $\overleftarrow{d}[h]$ by a generalised Taylor expansion. As an illustrative example we consider the differential maps corresponding to the quantisation of quasitriangular commutator Lie bialgebras of [Hudson and Pulmannová, Lett. Math. Phys. 72 (2005)].
\end{abstract}

1. Introduction. Motivated by quantum stochastic calculus [5], in which case the multiplication rule is essentially that for iterated stochastic integrals and the natural coproduct is related to the continuous tensor product structure of Fock space, in [6] we introduced a noncommutative generalisation of the shuffle product Hopf algebra, called the Itô Hopf algebra. The universal enveloping algebra of the commutator Lie algebra of the underlying associative algebra is embedded in this as a Hopf subalgebra. Furthermore, we showed in [7] that this could be used to effect a quantisation of commutator Lie bialgebras of quasitriangular [2] type. The quantisation is effected by conjugating the standard coproduct by a "double product integral" whose generator is derived from the solution of the classical Yang-Baxter equation determining the quasitriangular structure, to obtain a deformed coproduct.

2000 Mathematics Subject Classification: Primary 16W30.

Key words and phrases: Hopf algebra, deformed coproduct, deformed differential map, quantisation.

This work was begun when the first author was visiting the University of Łódź in 2002 and completed when the second author visited Nottingham Trent University in 2005, supported by the London Mathematical Society. We are grateful for the generosity and hospitality of these institutions.

SP was supported by Center of excellence SAS, CEPI I/2/2005, grant APVV- 0071-06 and grant VEGA 2/6088/26. 
The purpose of this article is to investigate more general deformed coproducts, and corresponding quantisations, living in the generalised shuffle product Hopf algebra, which are not given by such conjugations. We shall investigate them using the corresponding differential maps. These were introduced in [6] in the case of the undeformed coproduct where they were found to be useful in the construction of double product integrals. Here we show that deformations of the differential maps of [6] can be associated with deformations of the coproduct. Corresponding to the multiplicativity, coassociativity and counitality of the deformed coproduct, the differential maps satisfy respectively the Leibniz-Itô formula, a mutual commutativity condition and a counitality condition related to an analog of the Maclaurin expansion for polynomials. Conversely, given deformed differential maps satisfying these three conditions, a corresponding deformed coproduct can be constructed by an analog of the Taylor expansion.

In a subsequent paper we shall show how such differential maps can be constructed, essentially from an infinitesimal which is the first order coefficient in their formal power series expansions, thus giving a general method of quantising commutator Lie bialgebras.

We use the following notational convention. If $\mathcal{V}_{1}, \ldots, \mathcal{V}_{n}$ are vector spaces and $\pi$ is a permutation of $\{1, \ldots, n\}$ then $\tau_{\pi}$ is the linear map from $\mathcal{V}_{1} \otimes \cdots \otimes \mathcal{V}_{n}$ to $\mathcal{V}_{\pi(1)} \otimes \cdots \otimes \mathcal{V}_{\pi(n)}$ which appropriately permutes the components of product tensors.

2. The Itô Hopf algebra. Let $\mathcal{L}$ be a not necessarily unital associative algebra over a field $\mathbb{F}$ and let $\mathcal{T}=\bigoplus_{n=0}^{\infty} \otimes^{n} \mathcal{L}$ denote the vector space of all tensors over $\mathcal{L}$. Thus elements of $\mathcal{T}$ are sequences $T=\left(T_{0}, T_{1}, T_{2}, \ldots\right)$ of homogeneous tensors $T_{n} \in \bigotimes^{n} \mathcal{L}$ of which only finitely many are nonzero. $\mathcal{T}$ becomes a unital associative algebra under the multiplication $R S=T$, where the homogeneous component $T_{n}$ of rank $n$ of the tensor $T$ is defined in terms of those of $R$ and $S$ by

$$
T_{n}=\sum_{A \cup B=\{1, \ldots, n\}} R_{|A|}^{A} S_{|B|}^{B},
$$

where the sum is over all $3^{n}$ ordered pairs of subsets $(A, B)$ whose union is $\{1, \ldots, n\}, R_{|A|}^{A}$ indicates that the $|A|$ th rank homogeneous component $R_{|A|}$ of $R$ is placed in the tensor product $\bigotimes_{j \in A} \mathcal{L}$ of the copies of $\mathcal{L}$ labelled by elements $j$ of $A$ within the full $n$-fold tensor product $\otimes^{n} \mathcal{L}, S_{|B|}^{B}$ is defined analogously, so that $R_{|A|}^{A}$ and $S_{|B|}^{B}$ jointly occupy every copy of $\mathcal{L}$ in $\bigotimes^{n} \mathcal{L}$, and double occupancies are reduced using the multiplication in $\mathcal{L}$. Note that when the multiplication in $\mathcal{L}$ is trivial, all products vanishing, then the associative algebra $\mathcal{T}$ is just the shuffle algebra over $\mathcal{L}$. 
The algebra $\mathcal{T}$ becomes a Hopf algebra when the coproduct $\Delta_{0}$ is defined by linear extension of the action on homogeneous product tensors

$$
\Delta_{0}\left(L_{1} \otimes \cdots \otimes L_{n}\right)=\sum_{j=0}^{n}\left(L_{1} \otimes \cdots \otimes L_{j}\right) \otimes\left(L_{j+1} \otimes \cdots \otimes L_{n}\right) .
$$

The counit $\varepsilon$ is

$$
\varepsilon:\left(T_{0}, T_{1}, T_{2}, \ldots\right) \mapsto T_{0}
$$

and the antipode $S$ acts on homogeneous product tensors as

$$
S\left(L_{1} \otimes \cdots \otimes L_{n}\right)=(-1)^{n} L_{n} \otimes \cdots \otimes L_{1}+\text { terms of lower rank. }
$$

An explicit formula for $S$ is given in the Appendix.

The kernel of $\varepsilon$ is evidently the ideal $\mathcal{T}_{0}$ of $\mathcal{T}$ comprising tensors whose zero-rank homogeneous component is zero. We call the map

$$
\varepsilon_{1}: \mathcal{T}_{0} \ni\left(0, T_{1}, T_{2}, \ldots\right) \mapsto T_{1} \in \mathcal{L}
$$

the enabling map. Note that it is a homomorphism of associative algebras.

The map $\mathcal{L} \ni L \mapsto(0, L, 0,0, \ldots) \in \mathcal{T}$ is a Lie algebra homomorphism when both $\mathcal{L}$ and $\mathcal{T}$ have their commutator Lie brackets, whose universal extension is an isomorphism of Hopf algebras from the universal enveloping algebra $\mathcal{U}$ of $\mathcal{L}$ onto the Hopf subalgebra $\mathcal{S}$ of $\mathcal{T}$ consisting of symmetric tensors.

3. The undeformed differential maps. We define right and left differential maps $\vec{d}_{0}$ and $\overleftarrow{d}_{0}$ from $\mathcal{T}$ to $\mathcal{T} \otimes \mathcal{L}$ and $\mathcal{L} \otimes \mathcal{T}$ respectively by linear extension of the actions

$$
\begin{aligned}
& \vec{d}_{0}\left(1_{\mathcal{T}}\right)=0, \quad \vec{d}_{0}\left(L_{1} \otimes \cdots \otimes L_{n}\right)=\left(L_{1} \otimes \cdots \otimes L_{n-1}\right) \otimes L_{n}, \\
& \overleftarrow{d}_{0}\left(1_{\mathcal{T}}\right)=0, \quad \overleftarrow{d}_{0}\left(L_{1} \otimes \cdots \otimes L_{n}\right)=L_{1} \otimes\left(L_{2} \otimes \cdots \otimes L_{n}\right),
\end{aligned}
$$

respectively, for arbitrary $n \in \mathbb{N}$ and $L_{1}, \ldots, L_{n} \in \mathcal{L}$. Alternatively

$$
\begin{aligned}
& \vec{d}_{0}(T)=\left(\operatorname{id}_{\mathcal{T}} \otimes \varepsilon_{1}\right)\left(\Delta_{0}(T)-T \otimes 1_{\mathcal{T}}\right), \\
& \overleftarrow{d}_{0}(T)=\left(\varepsilon_{1} \otimes \operatorname{id}_{\mathcal{T}}\right)\left(\Delta_{0}(T)-1_{\mathcal{T}} \otimes T\right)
\end{aligned}
$$

for arbitrary $T \in \mathcal{T}$. As a consequence of the latter form, they both satisfy the Leibniz-Itô formula

$$
\begin{aligned}
& \vec{d}_{0}(S T)=\vec{d}_{0}(S) T+S \vec{d}_{0}(T)+\vec{d}_{0}(S) \vec{d}_{0}(T), \\
& \overleftarrow{d}_{0}(S T)=\overleftarrow{d}_{0}(S) T+S \overleftarrow{d}_{0}(T)+\overleftarrow{d}_{0}(S) \overleftarrow{d}_{0}(T)
\end{aligned}
$$

for arbitrary $S, T \in \mathcal{T}$, where, in the first two terms of the right hand sides, $\mathcal{T} \otimes \mathcal{L}$ and $\mathcal{L} \otimes \mathcal{T}$ are regarded as $\mathcal{T}$-modules with the natural multiplicative 
actions and, in the third terms, as associative algebras using the tensor product multiplication $\left({ }^{1}\right)$.

For $T \in \mathcal{T}$, if both $\vec{d}_{0}(T)=0$ and $\varepsilon(T)=0$, then $T=0$. Similarly if, for $T \in \mathcal{T} \otimes \mathcal{T}$, both

$$
\left(\operatorname{id}_{\mathcal{T}} \otimes \vec{d}_{0}\right) T=0, \quad\left(\operatorname{id}_{\mathcal{T}} \otimes \varepsilon\right) T=0,
$$

it follows that $T=0$.

By checking actions on homogeneous product tensors it can be verified that the differential maps satisfy the commutation relation

$$
\left(\operatorname{id}_{\mathcal{L}} \otimes \vec{d}_{0}\right) \overleftarrow{d}_{0}=\left(\overleftarrow{d}_{0} \otimes \mathrm{id}_{\mathcal{L}}\right) \vec{d}_{0}
$$

and that, in so far as

$$
\mathcal{T} \otimes \mathcal{T}=\bigoplus_{n=0}^{\infty}\left(\mathcal{T} \otimes\left(\otimes^{n} \mathcal{L}\right)\right)=\bigoplus_{n=0}^{\infty}\left(\left(\otimes^{n} \mathcal{L}\right) \otimes \mathcal{T}\right)
$$

the Taylor expansions

$$
\Delta_{0}=\bigoplus_{n=0}^{\infty} \vec{d}_{0}^{(n)}=\bigoplus_{n=0}^{\infty} \overleftarrow{d}_{0}^{(n)}
$$

hold, where the iterated differential maps are defined by $\vec{d}_{0}^{(0)}=\overleftarrow{d}_{0}^{(0)}=\mathrm{id} \mathcal{T}$, $\vec{d}_{0}^{(1)}=\vec{d}_{0}, \overleftarrow{d}_{0}^{(1)}=\overleftarrow{d}_{0}$ and, for $n>1$,

$$
\vec{d}_{0}^{(n)}=\left(\vec{d}_{0} \otimes \mathrm{id}_{\otimes^{n-1} \mathcal{L}}\right) \vec{d}_{0}^{(n-1)}, \quad \overleftarrow{d}_{0}^{(n)}=\left(\mathrm{id}_{\otimes^{n-1} \mathcal{L}} \otimes \overleftarrow{d}_{0}\right) \overleftarrow{d}_{0}^{(n-1)} .
$$

Also we have the Maclaurin formulas

$$
\left(\varepsilon \otimes \operatorname{id}_{\otimes^{n} \mathcal{L}}\right) \vec{d}_{0}^{(n)}=\left(\operatorname{id}_{\otimes^{n} \mathcal{L}} \otimes \varepsilon\right) \overleftarrow{d}_{0}^{(n)}
$$

both being equal to the projection

$$
\mathcal{T} \ni\left(T_{0}, T_{1}, T_{2}, \ldots\right) \mapsto T_{n} \in \otimes^{n} \mathcal{L} .
$$

We note that $\Delta_{0}$ is the unique solution of the "differential equation", for a linear map from $\mathcal{T}$ to $\mathcal{T} \otimes \mathcal{T}$,

$$
\left(\mathrm{id}_{\mathcal{T}} \otimes \vec{d}_{0}\right) \Delta_{0}=\left(\Delta_{0} \otimes \mathrm{id}_{\mathcal{L}}\right) \vec{d}_{0}, \quad\left(\mathrm{id}_{\mathcal{T}} \otimes \varepsilon\right) \Delta_{0}=\mathrm{id}_{\mathcal{T}}
$$

Indeed, that it satisfies (7) follows from the corresponding Taylor expansion. To prove uniqueness, suppose that $\Delta_{0}^{\prime}$ also satisfies (7). Then the difference $D=\Delta_{0}-\Delta_{0}^{\prime}$ is a linear map from $\mathcal{T}$ to $\mathcal{T} \otimes \mathcal{T}$ satisfying

$$
\left(\operatorname{id}_{\mathcal{T}} \otimes \vec{d}_{0}\right) D=\left(D \otimes \operatorname{id}_{\mathcal{L}}\right) \vec{d}_{0}, \quad\left(\operatorname{id}_{\mathcal{T}} \otimes \varepsilon\right) D=0 .
$$

Since $\vec{d}_{0}\left(1_{\mathcal{T}}\right)=0$ we deduce that

$$
\left(\operatorname{id}_{\mathcal{T}} \otimes \vec{d}_{0}\right) D\left(1_{\mathcal{T}}\right)=\left(D \otimes \operatorname{id}_{\mathcal{L}}\right) \vec{d}_{0}\left(1_{\mathcal{T}}\right)=0, \quad\left(\operatorname{id}_{\mathcal{T}} \otimes \varepsilon\right) D\left(1_{\mathcal{T}}\right)=0
$$

$\left({ }^{1}\right)$ By modifying either the right or the left actions by the inclusion of the third term, it can be seen that both the right and left differential maps define differential calculi in the sense of Woronowicz [8], [4]. 
and hence from $(6)$ that $D\left(1_{\mathcal{T}}\right)=0$. Next consider a rank 1 tensor $(0, L, 0,0, \ldots) \in \mathcal{T}$. From $(7)$ we deduce that

$$
\begin{aligned}
\left(\operatorname{id}_{\mathcal{T}} \otimes \vec{d}_{0}\right) D(0, L, 0,0, \ldots) & =\left(D \otimes \operatorname{id}_{\mathcal{L}}\right) \vec{d}_{0}(0, L, 0,0, \ldots) \\
& =\left(D \otimes \operatorname{id}_{\mathcal{L}}\right)\left(1_{\mathcal{T}} \otimes L\right)=0
\end{aligned}
$$

since $D\left(1_{\mathcal{T}}\right)=0$, whence, since also $\left(\mathrm{id}_{\mathcal{T}} \otimes \varepsilon\right) D(0, L, 0,0, \ldots)=0$, it again follows from (6) that $D(0, L, 0,0, \ldots)=0$. A simple inductive argument shows that similarly $D$ vanishes on homogeneous tensors of arbitrary rank.

A similar argument shows that $\Delta_{0}$ is the unique solution of

$$
\left(\overleftarrow{d}_{0} \otimes \mathrm{id}_{\mathcal{T}}\right) \Delta_{0}=\left(\operatorname{id}_{\mathcal{L}} \otimes \Delta_{0}\right) \overleftarrow{d}_{0}, \quad\left(\varepsilon \otimes \operatorname{id}_{\mathcal{T}}\right) \Delta_{0}=\operatorname{id}_{\mathcal{T}} .
$$

4. From deformed coproducts to differential maps. We equip the space $\mathcal{T}[[h]]$ of formal power series with coefficients in $\mathcal{T}$ with the convolution product derived from that in $\mathcal{T}$,

$$
\sum_{N=0}^{\infty} h^{N} S^{(N)} \sum_{N=0}^{\infty} h^{N} T^{(N)}=\sum_{N=0}^{\infty} h^{N} \sum_{j=0}^{N} S^{(j)} T^{(N-j)} .
$$

We denote by $\mathcal{A}$ the subalgebra of $\mathcal{T}[[h]]$ consisting of formal power series $\sum_{N=0}^{\infty} h^{N} S^{(N)}$ whose zero-order coefficient $S^{(0)}$ is a symmetric tensor. Thus the universal enveloping algebra $\mathcal{U} \simeq \mathcal{S}$ is embedded as the algebra of "constants" in $\mathcal{A}$ and we can seek quantum groups, in the form of deformations of $\mathcal{U}$, in $\mathcal{A}$. We do this by equipping $\mathcal{T}$ with a deformation coproduct

$$
\Delta[h]=\sum_{N=0}^{\infty} h^{N} \Delta_{N}: \mathcal{T} \rightarrow(\mathcal{T} \otimes \mathcal{T})[[h]],
$$

where the zero-order coefficient $\Delta_{0}$ is the natural coproduct defined in $\mathcal{T}$ by (2). We shall assume that the undeformed counit $\varepsilon$ in $\mathcal{T}$ remains counital for the deformed coproduct;

$$
\left(\varepsilon \otimes \operatorname{id}_{\mathcal{T}}\right) \Delta[h]=\left(\operatorname{id}_{\mathcal{T}} \otimes \varepsilon\right) \Delta[h]=\operatorname{id}_{\mathcal{T}} .
$$

Here and elsewhere, maps defined on coefficient algebras of formal power series are extended to formal power series by actions on coefficients. In particular, the coefficients of order greater than zero of $\Delta[h]$ are annihilated by $\varepsilon$ :

$$
\left(\varepsilon \otimes \operatorname{id}_{\mathcal{T}}\right) \Delta_{N}=\left(\operatorname{id}_{\mathcal{T}} \otimes \varepsilon\right) \Delta_{N}=0, \quad N=1,2, \ldots
$$

We associate with the deformed coproduct $\Delta[h]$ the right and left deformed differential maps $\vec{d}[h]: \mathcal{T} \rightarrow(\mathcal{T} \otimes \mathcal{L})[[h]], \overleftarrow{d}[h]: \mathcal{T} \rightarrow(\mathcal{L} \otimes \mathcal{T})[[h]]$ defined by the generalisations of $(5)$ :

$$
\begin{aligned}
& \vec{d}[h](T)=\left(\operatorname{id}_{\mathcal{T}} \otimes \varepsilon_{1}\right)\left(\Delta[h](T)-T \otimes 1_{\mathcal{T}}\right), \\
& \overleftarrow{d}[h](T)=\left(\varepsilon_{1} \otimes \operatorname{id}_{\mathcal{T}}\right)\left(\Delta[h](T)-1_{\mathcal{T}} \otimes T\right) .
\end{aligned}
$$


Note that in view of $(8)$,

$$
\left(\operatorname{id}_{\mathcal{T}} \otimes \varepsilon\right)\left(\Delta[h](T)-T \otimes 1_{\mathcal{T}}\right)=T-\left(\operatorname{id}_{\mathcal{T}} \otimes \varepsilon\right)\left(T \otimes 1_{\mathcal{T}}\right)=0
$$

so that $\Delta[h](T)-T \otimes 1_{\mathcal{T}}$ belongs to the domain of $\operatorname{id}_{\mathcal{T}} \otimes \varepsilon_{1}$ and so $\vec{d}[h](T)$, and similarly $\overleftarrow{d}[h](T)$, are well defined. Also

$$
\vec{d}[h]=\sum_{N=0}^{\infty} h^{N} \vec{d}_{N}, \quad \overleftarrow{d}[h]=\sum_{N=0}^{\infty} h^{N} \overleftarrow{d}_{N},
$$

where the zero-order coefficients $\vec{d}_{0}$ and $\overleftarrow{d}_{0}$ are the undeformed differential maps. In view of $(8)$,

$$
\begin{aligned}
\left(\varepsilon \otimes \operatorname{id}_{\mathcal{L}}\right) \vec{d}[h](T) & =\left(\varepsilon \otimes \operatorname{id}_{\mathcal{L}}\right)\left(\operatorname{id}_{\mathcal{T}} \otimes \varepsilon_{1}\right)\left(\Delta[h](T)-T \otimes 1_{\mathcal{T}}\right) \\
& =\varepsilon_{1}\left(\varepsilon \otimes \operatorname{id}_{\mathcal{T}}\right)\left(\Delta[h](T)-T \otimes 1_{\mathcal{T}}\right)=\varepsilon_{1}\left(T-\varepsilon(T) 1_{\mathcal{T}}\right),
\end{aligned}
$$

and a similar argument shows that

$$
\left(\operatorname{id}_{\mathcal{L}} \otimes \varepsilon\right) \overleftarrow{d}[h](T)=\varepsilon_{1}\left(T-\varepsilon(T) 1_{\mathcal{T}}\right)
$$

In particular,

$$
\left(\varepsilon \otimes \operatorname{id}_{\mathcal{L}}\right) \vec{d}[h]=\left(\operatorname{id}_{\mathcal{L}} \otimes \varepsilon\right) \overleftarrow{d}[h]
$$

and

$$
\left(\varepsilon \otimes \operatorname{id}_{\mathcal{L}}\right) \vec{d}_{N}=\left(\operatorname{id}_{\mathcal{L}} \otimes \varepsilon\right) \overleftarrow{d}_{N}=0, \quad N=1,2, \ldots
$$

THEOREM 1. Assume that the deformed coproduct $\Delta[h]$ is multiplicative:

$$
\Delta[h](S T)=\Delta[h](S) \Delta[h](T) .
$$

Then the corresponding deformed differential maps $\vec{d}[h]$ and $\overleftarrow{d}[h]$ satisfy the Leibniz-Itô formulas

$$
\begin{aligned}
& \vec{d}[h](S T)=\vec{d}[h](S) T+S \vec{d}[h](T)+\vec{d}[h](S) \vec{d}[h](T), \\
& \overleftarrow{d}[h](S T)=\overleftarrow{d}[h](S) T+S \overleftarrow{d}[h](T)+\overleftarrow{d}[h](S) \overleftarrow{d}[h](T)
\end{aligned}
$$

Here $(\mathcal{T} \otimes \mathcal{L})[[h]]$ and $(\mathcal{L} \otimes \mathcal{T})[[h]]$ are regarded as $\mathcal{T}$-bimodules by means of the natural multiplicative actions on coefficients and as algebras using the convolution product for formal power series based on the tensor product multiplication for the coefficients.

Proof. Using the definition of $\vec{d}[h]$, the multiplicativity of the maps $\Delta[h]$ and $\operatorname{id}_{\mathcal{T}} \otimes \varepsilon_{1}$, and the forms of the left and right actions of $\mathcal{T}$ on $(\mathcal{T} \otimes \mathcal{L})[[h]]$, we have

$$
\begin{aligned}
\vec{d}[h](S) \vec{d}[h](T) & \left(\left(\operatorname{id}_{\mathcal{T}} \otimes \varepsilon_{1}\right)\left(\Delta[h](S)-S \otimes 1_{\mathcal{T}}\right)\right)\left(\left(\operatorname{id}_{\mathcal{T}} \otimes \varepsilon_{1}\right)\left(\Delta[h](T)-T \otimes 1_{\mathcal{T}}\right)\right) \\
= & \left(\operatorname{id}_{\mathcal{T}} \otimes \varepsilon_{1}\right)\left(\left(\Delta[h](S)-S \otimes 1_{\mathcal{T}}\right)\left(\Delta[h](T)-T \otimes 1_{\mathcal{T}}\right)\right) \\
= & \left(\operatorname{id}_{\mathcal{T}} \otimes \varepsilon_{1}\right)\left(\Delta[h](S) \Delta[h](T)-\Delta[h](S)\left(V \otimes 1_{\mathcal{T}}\right)-\left(S \otimes 1_{\mathcal{T}}\right) \Delta[h](T) .\right. \\
& \left.+\left(S \otimes 1_{\mathcal{T}}\right)\left(T \otimes 1_{\mathcal{T}}\right)\right)
\end{aligned}
$$




$$
\begin{aligned}
= & \left(\operatorname{id}_{\mathcal{U}} \otimes \varepsilon_{1}\right)\left(\Delta[h](S T)-(S T) \otimes 1_{\mathcal{T}}-\left(\Delta[h](S)-S \otimes 1_{\mathcal{T}}\right)\left(T \otimes 1_{\mathcal{T}}\right) .\right. \\
& \left.-\left(S \otimes 1_{\mathcal{T}}\right)\left(\Delta[h](T)-T \otimes 1_{\mathcal{T}}\right)\right) \\
= & \vec{d}[h](S T)-(\vec{d}[h](S)) T-S(\vec{d}[h](T)),
\end{aligned}
$$

from which the Leibniz-Itô formula for $\vec{d}[h]$ follows. That for $\overleftarrow{d}[h]$ is proved similarly.

Theorem 2. Assume that the deformed coproduct $\Delta[h]$ is coassociative:

$$
\left(\mathrm{id}_{\mathcal{T}} \otimes \Delta[h]\right) \Delta[h]=\left(\Delta[h] \otimes \mathrm{id}_{\mathcal{T}}\right) \Delta[h] .
$$

Then $\vec{d}[h]$ and $\overleftarrow{d}[h]$ satisfy the commutativity condition

$$
\left(\operatorname{id}_{\mathcal{L}} \otimes \vec{d}[h]\right) \overleftarrow{d}[h]=\left(\overleftarrow{d}[h] \otimes \operatorname{id}_{\mathcal{L}}\right) \vec{d}[h] .
$$

Here formal power series whose coefficients are maps are composed by convolution, for example

$$
\left(\operatorname{id}_{\mathcal{T}} \otimes \Delta[h]\right) \Delta[h]=\sum_{N=0}^{\infty} h^{N} \sum_{j=0}^{N}\left(\operatorname{id}_{\mathcal{T}} \otimes \Delta_{j}\right) \Delta_{N-j} .
$$

Proof. For arbitrary $T \in \mathcal{T}$,

$$
\begin{aligned}
\left(\left(\operatorname{id}_{\mathcal{L}} \otimes \vec{d}[h]\right)\right. & \overleftarrow{d}[h])(T)=\left(\operatorname{id}_{\mathcal{L}} \otimes \vec{d}[h]\right)(\overleftarrow{d}[h](T)) \\
= & \left(\operatorname{id}_{\mathcal{L}} \otimes \vec{d}[h]\right)\left(\left(\varepsilon_{1} \otimes \mathrm{id}_{\mathcal{T}}\right)\left(\Delta[h](T)-1_{\mathcal{T}} \otimes T\right)\right) \\
= & \left(\left(\operatorname{id}_{\mathcal{L}} \otimes \vec{d}[h]\right)\left(\varepsilon_{1} \otimes \mathrm{id}_{\mathcal{T}}\right)\right)\left(\Delta[h](T)-1_{\mathcal{T}} \otimes T\right) \\
= & \left(\varepsilon_{1} \otimes \vec{d}[h]\right)\left(\Delta[h](T)-1_{\mathcal{T}} \otimes T\right) \\
= & \left(\left(\varepsilon_{1} \otimes \operatorname{id}_{T \otimes \mathcal{L}}\right)\left(\operatorname{id}_{\mathcal{T}} \otimes \vec{d}[h]\right)\right)\left(\Delta[h](T)-1_{\mathcal{T}} \otimes T\right) \\
= & \left(\varepsilon_{1} \otimes \operatorname{id}_{T \otimes \mathcal{L}}\right)\left(\operatorname{id}_{\mathcal{T}} \otimes \operatorname{id}_{\mathcal{T}} \otimes \varepsilon_{1}\right)\left\{\left(\operatorname{id}_{\mathcal{T}} \otimes \Delta[h]\right)\left(\Delta[h](T)-1_{\mathcal{T}} \otimes T\right)\right. \\
& \left.-1_{\mathcal{T}} \otimes\left(\Delta[h](T)-1_{\mathcal{T}} \otimes T\right)\right\} \\
= & \left(\varepsilon_{1} \otimes \mathrm{id}_{\mathcal{T}} \otimes \varepsilon_{1}\right)\left\{\left(\left(\mathrm{id}_{\mathcal{T}} \otimes \Delta[h]\right) \Delta[h]\right)(T)-1_{\mathcal{T}} \otimes \Delta[h](T)\right. \\
& \left.-\Delta[h](T) \otimes 1_{\mathcal{T}}+1_{\mathcal{T}} \otimes 1_{\mathcal{T}} \otimes T\right\} .
\end{aligned}
$$

A similar argument shows that

$$
\begin{aligned}
\left(\left(\overleftarrow{d}[h] \otimes \operatorname{id}_{\mathcal{L}}\right) \vec{d}[h]\right)(T) & \\
= & \left(\varepsilon_{1} \otimes \operatorname{id}_{\mathcal{T}} \otimes \varepsilon_{1}\right)\left\{\left(\left(\Delta[h] \otimes \operatorname{id}_{\mathcal{T}}\right) \Delta[h]\right)(T)-1_{\mathcal{T}} \otimes \Delta[h](T)\right. \\
& \left.-\Delta[h](T) \otimes 1_{\mathcal{T}}+1_{\mathcal{T}} \otimes 1_{\mathcal{T}} \otimes T\right\} .
\end{aligned}
$$

Comparing these two expressions we see that (13) follows from (12).

The next theorem shows that the deformed coproduct $\Delta[h]$ can be regarded as a "flow" generated by either the right or the left deformed differential map. 
TheOREM 3. Suppose that $\Delta[h]$ is coassociative. Then it is the unique solution of either of the "differential equations"

$$
\begin{array}{ll}
\left(\operatorname{id}_{\mathcal{T}} \otimes \vec{d}[h]\right) \Delta[h]=\left(\Delta[h] \otimes \operatorname{id}_{\mathcal{L}}\right) \vec{d}[h], & \left(\operatorname{id}_{\mathcal{T}} \otimes \varepsilon\right) \Delta[h]=\operatorname{id}_{\mathcal{T}}, \\
\left(\overleftarrow{d}[h] \otimes \operatorname{id}_{\mathcal{T}}\right) \Delta[h]=\left(\operatorname{id}_{\mathcal{L}} \otimes \Delta[h]\right) \overleftarrow{d}[h], & \left(\varepsilon \otimes \operatorname{id}_{\mathcal{T}}\right) \Delta[h]=\operatorname{id}_{\mathcal{T}} .
\end{array}
$$

Proof. Let us prove that $\Delta[h]$ is the unique solution of (14); the case of (15) is proved similarly. To prove that it satisfies (14), using the definition of $\vec{d}[h]$ and the coassociativity of $\Delta[h]$ we have, for arbitrary $T \in \mathcal{T}$,

$$
\begin{aligned}
& \left(\left(\operatorname{id}_{\mathcal{T}} \otimes \vec{d}[h]\right) \Delta[h]\right)(T) \\
& =\left(\operatorname{id}_{\mathcal{T}} \otimes \operatorname{id}_{\mathcal{T}} \otimes \varepsilon_{1}\right)\left(\left(\left(\operatorname{id}_{\mathcal{T}} \otimes \Delta[h]\right) \Delta[h]\right)(T)-\tau_{(1,3,2)}\left(1_{\mathcal{T}} \otimes \Delta[h](T)\right)\right) \\
& =\left(\operatorname{id}_{\mathcal{T}} \otimes \operatorname{id}_{\mathcal{T}} \otimes \varepsilon_{1}\right)\left(\left(\left(\Delta[h] \otimes \operatorname{id}_{T}\right) \Delta[h]\right)(T)-\tau_{(1,3,2)}\left(1_{\mathcal{T}} \otimes \Delta[h](T)\right)\right) \\
& =\left(\Delta[h] \otimes \operatorname{id}_{\mathcal{L}}\right)\left(\left(\operatorname{id}_{\mathcal{T}} \otimes \varepsilon_{1}\right)\left(\Delta[h](T)-1_{\mathcal{T}} \otimes T\right)\right) \\
& =\left(\Delta[h] \otimes \operatorname{id}_{\mathcal{L}}\right)(\vec{d}[h](T))=\left(\left(\Delta[h] \otimes \operatorname{id}_{\mathcal{L}}\right) \vec{d}[h]\right)(T) .
\end{aligned}
$$

Also, by $(8),\left(\operatorname{id}_{\mathcal{T}} \otimes \varepsilon\right) \Delta[h](T)=T$. Hence (14) holds.

Let us now prove uniqueness. Equating coefficients of powers of $h$ in (14) we see that the zero-order coefficient $\Delta_{0}$ is the solution of (7) which is unique. The first order coefficient $\Delta_{1}$ satisfies

$$
\left(\operatorname{id}_{\mathcal{T}} \otimes \vec{d}_{0}\right) \Delta_{1}+\left(\operatorname{id}_{\mathcal{T}} \otimes \vec{d}_{1}\right) \Delta_{0}=\left(\Delta_{0} \otimes \operatorname{id}_{\mathcal{L}}\right) \vec{d}_{1}+\left(\Delta_{1} \otimes \operatorname{id}_{\mathcal{L}}\right) \vec{d}_{0},
$$

together with $\left(\operatorname{id}_{\mathcal{T}} \otimes \varepsilon\right) \Delta_{1}=0$. Suppose that both $\Delta_{1}$ and $\Delta_{1}^{\prime}$ satisfy these equations. Then their difference $D_{1}$ satisfies

$$
\left(\operatorname{id}_{\mathcal{T}} \otimes \vec{d}_{0}\right) D_{1}=\left(D_{1} \otimes \mathrm{id}_{\mathcal{L}}\right) \vec{d}_{0}, \quad\left(\operatorname{id}_{\mathcal{T}} \otimes \varepsilon\right) D_{1}=0 .
$$

But by the uniqueness of the solution to (7) such a map $D_{1}$ must vanish and hence the first order coefficient is unique. A similar inductive argument now shows that the $N$ th order coefficient $\Delta_{N}$ is unique.

5. From differential maps to deformed coproducts. Now let $\vec{d}[h]$ and $\overleftarrow{d}[h]$ be arbitrary maps of the form $(10)$ from $\mathcal{T}$ to $(\mathcal{T} \otimes \mathcal{L})[[h]]$ and $(\mathcal{L} \otimes \mathcal{T})[[h]]$ respectively. Our strategy in what follows is to construct a corresponding deformation coproduct $\Delta[h]$ by means of Taylor expansions

$$
\Delta[h]=\bigoplus_{n=0}^{\infty} \vec{d}^{(n)}[h]=\bigoplus_{n=0}^{\infty} \overleftarrow{d}^{(n)}[h]
$$

in terms of iterations of the maps $\vec{d}[h]$ and $\overleftarrow{d}[h]$. Our first task is to ensure that these sums are well defined formal power series. 
For the moment we assume only that (10) holds, that the coefficients $\overrightarrow{d_{0}}$ and $\overleftarrow{d}_{0}$ of $h^{0}$ are indeed the undeformed right and left differential maps, and that the higher order coefficients satisfy (11) for $N>0$. We denote by $\vec{d}^{(n)}[h]$ and $\overleftarrow{d}^{(n)}[h]$ the $n$-fold iterates

$$
\begin{aligned}
& \left(\vec{d}[h] \otimes\left(\otimes^{n-1} \operatorname{id}_{\mathcal{L}}\right)\right)\left(\vec{d}[h] \otimes\left(\bigotimes^{n-2} \operatorname{id}_{\mathcal{L}}\right)\right) \cdots \vec{d}[h], \\
& \left(\left(\bigotimes^{n-1} \operatorname{id}_{\mathcal{L}}\right) \otimes \overleftarrow{d}[h]\right)\left(\left(\bigotimes^{n-2} \operatorname{id}_{\mathcal{L}}\right) \otimes \overleftarrow{d}[h]\right) \cdots \overleftarrow{d}[h]
\end{aligned}
$$

formed by multiple convolution; it is convenient also to define $\vec{d}^{(0)}[h]=$ $\overleftarrow{d}^{(0)}[h]=\operatorname{id}_{\mathcal{T}}$.

For fixed $T \in \mathcal{T}$ consider the sequence of formal power series $\left(T_{0}[h], T_{1}[h]\right.$, $\left.T_{2}[h], \ldots\right)$ in which $T_{n}[h]=\vec{d}^{(n)}[h](T)$. The coefficients of $h^{0}$ in this sequence of formal power series consist of iterated actions of $\vec{d}_{0}$ on $T$. Because by (3) each action of $\vec{d}_{0}$ reduces by one the maximal nonzero rank of $T$, only finitely many of these coefficients are nonzero. More generally, the coefficient of $h^{N}$ in $T_{n}[h]$ is

$$
\sum_{j_{1}+\cdots+j_{n}=N}\left(\vec{d}_{j_{1}} \otimes\left(\otimes^{n-1} \operatorname{id}_{\mathcal{L}}\right)\right)\left(\vec{d}_{j_{2}} \otimes\left(\otimes^{n-2} \operatorname{id}_{\mathcal{L}}\right)\right) \cdots \vec{d}_{j_{n}}(T)
$$

where the sum is over ordered $n$-tuples of nonnegative integers $\left(j_{1}, \ldots, j_{n}\right)$ whose sum is $N$. For fixed $N$, a finite number of ordered $r$-tuples of positive integers $\left(k_{1}, \ldots, k_{r}\right)$ whose sum is $N$, that is, partitions of $N$, contribute to this sum, supplemented by $n-r$ zeros corresponding to actions of $\overrightarrow{d_{0}}$ which either fall entirely to the right of the actions of $\vec{d}_{k_{1}}, \ldots, \vec{d}_{k_{r}}$ or intercede between these actions, or fall entirely to the left of them. For each fixed partition, by the rank-reducing property of $\vec{d}_{0}$, only a finite number of actions of $\overrightarrow{d_{0}}$ may fall entirely to the right and yield a nonzero contribution to the coefficient of $h^{N}$ in $T_{n}[h]$. For the same reason, for each of these contributions, only a finite number of actions of $\vec{d}_{0}$ may lie between $\vec{d}_{k_{r}}$ and $\vec{d}_{k_{r-1}}$ and yield a nonzero contribution. Continuing this argument we see that the sequence $\left(T_{0}[h], T_{1}[h], T_{2}[h], \ldots\right)$ can be rearranged as a well defined element of $\left(\bigoplus_{n=0}^{\infty}\left(\mathcal{T} \otimes \otimes^{n} \mathcal{L}\right)\right)[[h]] \simeq(\mathcal{T} \otimes \mathcal{T})[[h]]$. We denote this by $\vec{\Delta}[h](T)$ and regard $\vec{\Delta}[h]$ as a map from $\mathcal{T}$ to the algebra $(\mathcal{T} \otimes \mathcal{T})[[h]]$. Note that by $(11)$,

$$
\begin{aligned}
\left(\varepsilon \otimes \mathrm{id}_{\mathcal{T}}\right) \vec{\Delta}[h](T) & =\left(\varepsilon \otimes \mathrm{id}_{\mathcal{T}}\right)\left(\vec{d}^{(0)}[h](T), \vec{d}^{(1)}[h](T), \vec{d}^{(2)}[h](T), \ldots\right) \\
& =\left(\varepsilon \otimes \operatorname{id}_{\mathcal{T}}\right)\left(\vec{d}_{0}^{(0)}(T), \vec{d}_{0}^{(1)}(T), \vec{d}_{0}^{(2)}(T), \ldots\right)=T .
\end{aligned}
$$

Also

$$
\begin{aligned}
\left(\operatorname{id}_{\mathcal{T}} \otimes \varepsilon\right) \vec{\Delta}[h](T) & =\left(\operatorname{id}_{\mathcal{T}} \otimes \varepsilon\right)\left(\vec{d}^{(0)}[h](T), \vec{d}^{(1)}[h](T), \vec{d}^{(2)}[h](T), \ldots\right) \\
& =\vec{d}^{(0)}[h](T)=T .
\end{aligned}
$$


In the same way, we may rearrange the sequence $\left(\overleftarrow{d}^{(0)}[h](T), \overleftarrow{d}^{(1)}[h](T)\right.$, $\left.\overleftarrow{d}^{(2)}[h](T), \ldots\right)$ to obtain a formal power series and hence a map $\overleftarrow{\Delta}[h]$ from $\mathcal{T}$ to $\left(\bigoplus_{n=0}^{\infty}\left(\otimes^{n} \mathcal{L} \otimes \mathcal{T}\right)\right)[[h]] \simeq(\mathcal{T} \otimes \mathcal{T})[[h]]$. In view of (16), (17) and the corresponding identities for $\bar{\Delta}[h]$ we have the counitality relations

$$
\begin{aligned}
\left(\varepsilon \otimes \operatorname{id}_{\mathcal{T}}\right) \vec{\Delta}[h] & =\left(\operatorname{id}_{\mathcal{T}} \otimes \varepsilon\right) \vec{\Delta}[h]=\left(\varepsilon \otimes \operatorname{id}_{\mathcal{T}}\right) \overleftarrow{\Delta}[h] \\
& =\left(\operatorname{id}_{\mathcal{T}} \otimes \varepsilon\right) \overleftarrow{\Delta}[h]=\operatorname{id}_{\mathcal{T}} .
\end{aligned}
$$

Evidently $\vec{\Delta}[h]$ and $\overleftarrow{\Delta}[h]$ satisfy (14) and (15) respectively for the given differential maps.

THEOREM 4. Suppose that $\vec{d}[h]$ (resp. $\overleftarrow{d}[h]$ ) satisfies the Leibniz-Itô formula. Then $\vec{\Delta}[h]$ (resp. $\overleftarrow{\Delta}[h])$ is multiplicative.

Proof. We give the proof for $\vec{\Delta}[h]$; that for the left map is similar. We shall show that, for arbitrary $S, T \in \mathcal{T}$, the difference

$$
P(S, T)[h]=\sum_{N=0}^{\infty} h^{N} P_{N}(S, T)=\vec{\Delta}[h](S T)-\vec{\Delta}[h](S) \vec{\Delta}[h](T)
$$

vanishes by proving by induction on $N$ that each $P_{N}(S, T)$ is 0 . We have $P_{0}(S, T)=0$ since the zero-order terms of $\vec{\Delta}[h](S T)$ and $\vec{\Delta}[h](S) \vec{\Delta}[h](T)$ are $\Delta(S T)$ and $\Delta(S) \Delta(T)$ respectively and the undeformed coproduct $\Delta$ is multiplicative.

Also, by multiplicativity of $\varepsilon$, for $N>1$,

$$
\begin{aligned}
& \left(\varepsilon \otimes \operatorname{id}_{\mathcal{T}}\right) P_{N}(S, T)=\left(\varepsilon \otimes \operatorname{id}_{\mathcal{T}}\right)\left(\vec{\Delta}_{N}(S T)-\sum_{j=0}^{N} \vec{\Delta}_{j}(S) \vec{\Delta}_{N-j}(T)\right) \\
& \quad=\left(\varepsilon \otimes \operatorname{id}_{\mathcal{T}}\right)\left(\vec{\Delta}_{N}(S T)\right)-\sum_{j=0}^{N}\left(\varepsilon \otimes \operatorname{id}_{\mathcal{T}}\right)\left(\vec{\Delta}_{j}(S)\right)\left(\varepsilon \otimes \operatorname{id}_{\mathcal{T}}\right)\left(\vec{\Delta}_{N-j}(T)\right)=0
\end{aligned}
$$

by (16). It follows from (6) that, in order to prove that $P_{N}(S, T)=0$, it is sufficient to show that

$$
\left(\overrightarrow{d_{0}} \otimes \operatorname{id}_{\mathcal{T}}\right) P_{N}(S, T)=0
$$

under the inductive assumption that $P_{0}(S, T)=P_{1}(S, T)=\cdots=P_{N-1}(S, T)$ $=0$.

Using the fact that the map $\operatorname{id}_{\mathcal{T}(\mathcal{L})} \otimes \vec{d}[h]$ from $\mathcal{T} \otimes \mathcal{T}$ to $(\mathcal{T} \otimes \mathcal{T} \otimes \mathcal{L})[[h]]$ satisfies the Leibniz-Itô formula when the algebra $(\mathcal{T} \otimes \mathcal{T} \otimes \mathcal{L})[[h]]$ is regarded as a $\mathcal{T} \otimes \mathcal{T}$-bimodule with the natural multiplicative actions, we have

$$
\begin{aligned}
\left(\operatorname{id}_{\mathcal{T}(\mathcal{L})} \otimes \vec{d}[h]\right) P(S, T) & {[h] } \\
& =\left(\operatorname{id}_{\mathcal{T}(\mathcal{L})} \otimes \vec{d}[h]\right)(\vec{\Delta}[h](S T)-\vec{\Delta}[h](S) \vec{\Delta}[h](T))
\end{aligned}
$$




$$
\begin{aligned}
= & \left(\operatorname{id}_{\mathcal{T}(\mathcal{L})} \otimes \vec{d}[h]\right)(\vec{\Delta}[h](S T))-\left(\operatorname{id}_{\mathcal{T}(\mathcal{L})} \otimes \vec{d}[h]\right)(\vec{\Delta}[h](S)) \vec{\Delta}[h](T) \\
& -\vec{\Delta}[h](S)\left(\operatorname{id}_{\mathcal{T}(\mathcal{L})} \otimes \vec{d}[h]\right)(\vec{\Delta}[h](T)) \\
& -\left(\operatorname{id}_{\mathcal{T}(\mathcal{L})} \otimes \vec{d}[h]\right)(\vec{\Delta}[h](S))\left(\operatorname{id}_{\mathcal{T}(\mathcal{L})} \otimes \vec{d}[h]\right)(\vec{\Delta}[h](T)) .
\end{aligned}
$$

Using (14) we may write this as

$$
\begin{aligned}
\left(\operatorname{id}_{\mathcal{T}(\mathcal{L})} \otimes \vec{d}[h]\right) & P(S, T)[h] \\
= & \left(\vec{\Delta}[h] \otimes \operatorname{id}_{\mathcal{L}}\right) \vec{d}[h](S T)-\left(\left(\vec{\Delta}[h] \otimes \operatorname{id}_{\mathcal{L}}\right) \vec{d}[h]\right)(S) \vec{\Delta}[h](T) \\
& -\vec{\Delta}[h](S)\left(\left(\vec{\Delta}[h] \otimes \operatorname{id}_{\mathcal{L}}\right) \vec{d}[h]\right)(T) \\
& -\left(\left(\vec{\Delta}[h] \otimes \operatorname{id}_{\mathcal{L}}\right) \vec{d}[h]\right)(S)\left(\left(\vec{\Delta}[h] \otimes \operatorname{id}_{\mathcal{L}}\right) \vec{d}[h]\right)(T),
\end{aligned}
$$

where now $(T \otimes \mathcal{T} \otimes \mathcal{L})[[h]]$ is a $\mathcal{T} \otimes \mathcal{T}$-module with multiplicative actions. Using the Leibniz-Itô formula again we may replace the first term on the right hand side of this expression by a sum of three terms which combine with the remaining three terms to give

$$
\begin{aligned}
\left(\operatorname{id}_{\mathcal{T}(\mathcal{L})} \otimes\right. & \vec{d}[h]) P(S, T)[h] \\
= & \left(\vec{\Delta}[h] \otimes \operatorname{id}_{\mathcal{L}}\right)(\vec{d}[h](S) T)-\left(\left(\vec{\Delta}[h] \otimes \operatorname{id}_{\mathcal{L}}\right) \vec{d}[h]\right)(S) \vec{\Delta}[h](T) \\
& +\left(\vec{\Delta}[h] \otimes \operatorname{id}_{\mathcal{L}}\right)(S \vec{d}[h](T))-\vec{\Delta}[h](S)\left(\left(\vec{\Delta}[h] \otimes \operatorname{id}_{\mathcal{L}}\right) \vec{d}[h]\right)(T) \\
& +\left(\vec{\Delta}[h] \otimes \operatorname{id}_{\mathcal{L}}\right)(\vec{d}[h](S) \vec{d}[h](T)) \\
& -\left(\vec{\Delta}[h] \otimes \operatorname{id}_{\mathcal{L}}\right)(\vec{d}[h](S))\left(\vec{\Delta}[h] \otimes \operatorname{id}_{\mathcal{L}}\right)(\vec{d}[h](T)) .
\end{aligned}
$$

Introducing a basis $\left(L_{1}, L_{2}, \ldots\right)$ of $\mathcal{L}$ and writing $\vec{d}[h](\cdot)=\sum_{j} d^{j}[h](\cdot) \otimes L_{j}$ where each $d^{j}[h]$ maps $\mathcal{T}$ to $\mathcal{T}[[h]]$, we may write this as

$$
\begin{aligned}
\left(\operatorname{id}_{\mathcal{T}(\mathcal{L})} \otimes\right. & \vec{d}[h]) P(S, T)[h] \\
= & \sum_{j} P\left(d^{j}[h](S), T\right)[h] \otimes L_{j}+\sum_{k} P\left(S, d^{k}[h](T)\right)[h] \otimes L_{k} \\
& \quad+\sum_{j, k} P\left(d^{j}[h](S), d^{k}[h](T)\right)[h] \otimes L_{j} L_{k},
\end{aligned}
$$

where $P\left(d^{j}[h](S), T\right)[h], P\left(S, d^{k}[h](T)\right)[h]$ and $P\left(d^{j}[h](S), d^{k}[h](T)\right)[h]$ are defined by convolution so that, for example,

$$
P\left(d^{j}[h](S), d^{k}[h](T)\right)[h]=\sum_{N=0}^{\infty} h^{N} \sum_{r+s+t=N} P_{r}\left(d_{s}^{j} S, d_{t}^{k} T\right),
$$

where $d^{j}[h]=\sum_{N=0}^{\infty} h^{N} d_{N}^{j}$ and the inner sum is over ordered triples $(r, s, t)$ of nonnegative integers whose sum is $N$. Let us now equate coefficients of 
$h^{N}$ on both sides of (19). In view of the inductive assumption we obtain

$$
\begin{aligned}
\left(\operatorname{id}_{\mathcal{T}(\mathcal{L})} \otimes \vec{d}[h]\right) P_{N}(S, T)= & \sum_{j} P_{N}\left(d_{0}^{j}(S), T\right) \otimes L_{j}+\sum_{k} P_{N}\left(S, d_{0}^{k}(T)\right) \otimes L_{k} \\
& +\sum_{j, k} P_{N}\left(d_{0}^{j}(S), d_{0}^{k}(T)\right) \otimes L_{j} L_{k} .
\end{aligned}
$$

Since $d_{0}^{j}$ and $d_{0}^{k}$ are the component maps of the undifferentiated differential map $\vec{d}_{0}$ in the chosen basis, they are rank reducing, and since

$$
P\left(1_{\mathcal{T}}, T\right)[h]=\vec{\Delta}[h](T)-\vec{\Delta}[h]\left(1_{\mathcal{T}}\right) \vec{\Delta}[h](T)=0,
$$

and similarly $P\left(S, 1_{\mathcal{T}}\right)[h]=0$, an induction on the degrees of $S$ and $T$ shows that $\left(\operatorname{id}_{\mathcal{T}(\mathcal{L})} \otimes \vec{d}[h]\right) P_{N}(S, T)$ and hence also $P_{N}(S, T)$ vanishes as required.

Next we show that, if $\vec{d}[h]$ and $\overleftarrow{d}[h]$ satisfy the commutativity condition (13) of Theorem 2, the maps $\vec{\Delta}[h]$ and $\overleftarrow{\Delta}[h]$ satisfy a modified coassociativity condition.

TheOrem 5. Suppose that $\vec{d}[h]$ and $\overleftarrow{d}[h]$ satisfy (13). Then

$$
\left(\operatorname{id}_{\mathcal{T}} \otimes \vec{\Delta}[h]\right) \overleftarrow{\Delta}[h]=\left(\overleftarrow{\Delta}[h] \otimes \operatorname{id}_{\mathcal{T}}\right) \vec{\Delta}[h] .
$$

Proof. (20) may be expressed informally as $\vec{\Delta}[h] \overleftarrow{\Delta}[h]=\overleftarrow{\Delta}[h] \vec{\Delta}[h]$ where appropriate ampliations are understood, and becomes intuitively clear when the commutatvity condition (13) is abbreviated similarly as $\vec{d}[h] \overleftarrow{d}[h]$ $=\overleftarrow{d}[h] \vec{d}[h]$ and $\vec{\Delta}[h]$ and $\overleftarrow{\Delta}[h]$ regarded as sums of powers of $\vec{d}[h]$ and $\overleftarrow{d}[h]$ respectively. More formally, we may expand the map $\left(\operatorname{id}_{\mathcal{T}} \otimes \vec{\Delta}[h]\right) \overleftarrow{\Delta}[h]$ from $\mathcal{T}$ to

$$
(\mathcal{T} \otimes \mathcal{T} \otimes \mathcal{T})[[h]]=\left(\bigoplus_{m, n=0}^{\infty}\left(\left(\otimes^{m} \mathcal{L}\right) \otimes \mathcal{T} \otimes\left(\otimes^{n} \mathcal{L}\right)\right)\right)[[h]]
$$

as

$$
\begin{aligned}
\left(\operatorname{id}_{\mathcal{T}} \otimes \vec{\Delta}[h]\right) \overleftarrow{\Delta}[h]= & \bigoplus_{m, n=0}^{\infty}\left\{\left(\left(\otimes^{n}\left(\operatorname{id}_{\mathcal{L}}\right)\right) \otimes\right.\right. \\
& \left.\left(\left(\vec{d}[h] \otimes\left(\otimes^{m-1} \operatorname{id}_{\mathcal{L}}\right)\right)\left(\vec{d}[h] \otimes\left(\otimes^{m-2} \operatorname{id}_{\mathcal{L}}\right)\right) \cdots \vec{d}[h]\right)\right) \\
& \left.\left(\left(\left(\bigotimes^{n-1} \operatorname{id}_{\mathcal{L}}\right) \otimes \overleftarrow{d}[h]\right)\left(\left(\bigotimes^{n-2} \operatorname{id}_{\mathcal{L}}\right) \otimes \overleftarrow{d}[h]\right) \cdots \overleftarrow{d}[h]\right)\right\} .
\end{aligned}
$$

By making repeated use of the commutativity condition (13), we have

$$
\begin{aligned}
& \left(\left(\otimes^{n} \operatorname{id}_{\mathcal{L}}\right) \otimes\left(\left(\vec{d}[h] \otimes\left(\otimes^{m-1} \operatorname{id}_{\mathcal{L}}\right)\right)\left(\vec{d}[h] \otimes\left(\bigotimes^{m-2} \operatorname{id}_{\mathcal{L}}\right)\right) \cdots \vec{d}[h]\right)\right) \\
& \left(\left(\left(\bigotimes^{n-1} \mathrm{id}_{\mathcal{L}}\right) \otimes \overleftarrow{d}[h]\right)\left(\left(\bigotimes^{n-2} \mathrm{id}_{\mathcal{L}}\right) \otimes \overleftarrow{d}[h]\right) \cdots \overleftarrow{d}[h]\right) \\
& =\left(\left(\left(\left(\bigotimes^{n-1} \mathrm{id}_{\mathcal{L}}\right) \otimes \overleftarrow{d}[h]\right)\left(\left(\bigotimes^{n-2} \mathrm{id}_{\mathcal{L}}\right) \otimes \overleftarrow{d}[h]\right) \cdots \overleftarrow{d}[h]\right)\right. \\
& \left.\otimes\left(\bigotimes^{m} \operatorname{id}_{\mathcal{L}}\right)\right)\left(\left(\vec{d}[h] \otimes\left(\bigotimes^{m-1} \operatorname{id}_{\mathcal{L}}\right)\right)\left(\vec{d}[h] \otimes\left(\bigotimes^{m-2} \operatorname{id}_{\mathcal{L}}\right)\right) \cdots \vec{d}[h]\right)
\end{aligned}
$$


as maps from $\mathcal{T}$ to $\left(\left(\otimes^{m} \mathcal{L}\right) \otimes \mathcal{T} \otimes\left(\otimes^{n} \mathcal{L}\right)\right)[[h]]$. Thus

$$
\begin{aligned}
\left(\operatorname{id}_{\mathcal{L}} \otimes\right. & \overleftarrow{\Delta}[h]) \vec{\Delta}[h] \\
= & \bigoplus_{m, n=0}^{\infty}\left\{\left(\left(\left(\otimes^{n-1} \operatorname{id}_{\mathcal{L}}\right) \otimes \overleftarrow{d}[h]\right)\left(\left(\otimes^{n-2} \operatorname{id}_{\mathcal{L}}\right) \otimes \overleftarrow{d}[h]\right) \cdots \overleftarrow{d}[h]\right)\right. \\
& \left.\left.\otimes\left(\otimes^{m}\left(\operatorname{id}_{\mathcal{L}}\right)\right)\right)\left(\left(\vec{d}[h] \otimes\left(\otimes^{m-1} \operatorname{id}_{\mathcal{L}}\right)\right)\left(\vec{d}[h] \otimes\left(\otimes^{m-2} \operatorname{id}_{\mathcal{L}}\right)\right) \cdots \vec{d}[h]\right)\right\} \\
= & \left(\overleftarrow{\Delta}[h] \otimes \operatorname{id}_{\mathcal{L}}\right) \vec{\Delta}[h] .
\end{aligned}
$$

Applying the map $\operatorname{id}_{\mathcal{T}} \otimes \varepsilon \otimes \mathrm{id}_{\mathcal{T}}$ to both sides of (20) and using (18) we finally obtain

$$
\begin{aligned}
\vec{\Delta}[h] & =\left(\operatorname{id}_{\mathcal{T}} \otimes \operatorname{id}_{\mathcal{T}}\right) \vec{\Delta}[h]=\left(\operatorname{id}_{\mathcal{T}} \otimes \varepsilon \otimes \operatorname{id}_{\mathcal{T}}\right)\left(\overleftarrow{\Delta}[h] \otimes \operatorname{id}_{\mathcal{T}}\right) \vec{\Delta}[h] \\
& =\left(\operatorname{id}_{\mathcal{T}} \otimes \varepsilon \otimes \operatorname{id}_{\mathcal{T}}\right)\left(\operatorname{id}_{\mathcal{T}} \otimes \vec{\Delta}[h]\right) \overleftarrow{\Delta}[h]=\left(\operatorname{id}_{\mathcal{T}} \otimes \operatorname{id}_{\mathcal{T}}\right) \overleftarrow{\Delta}[h]=\overleftarrow{\Delta}[h] .
\end{aligned}
$$

If we denote the common value of $\vec{\Delta}[h]$ and $\overleftarrow{\Delta}[h]$ by $\Delta[h]$, (20) now shows that $\Delta[h]$ is coassociative. This completes the construction of a deformation coproduct from the pair of differential maps $\vec{d}[h]$ and $\overleftarrow{d}[h]$.

6. An example. We consider a deformation coproduct of the quasitriangular type constructed in [7],

$$
\Delta_{0}[h](T)=R[h] \Delta_{0}(T) R[h]^{-1},
$$

where $R[h]=\vec{\prod}(1+d r[h])$ is the double product integral generated by the element $r[h]$ of $h(\mathcal{L} \otimes \mathcal{L})[[h]]$ satisfying the condition, sufficient for $R[h]$ to satisfy the quantum Yang-Baxter equation,

$$
\begin{aligned}
& r[h]^{1,2} r[h]^{1,3}+r[h]^{1,2} r[h]^{2,3}+r[h]^{1,3} r[h]^{2,3}+r[h]^{1,2} r[h]^{1,3} r[h]^{2,3} \\
& \quad=[h]^{1,3} r[h]^{1,2}+r[h]^{2,3} r[h]^{1,3}+r[h]^{2,3} r[h]^{1,2}+r[h]^{2,3} r[h]^{1,3} r[h]^{1,2},
\end{aligned}
$$

so that in particular the first order coefficient $r_{1}$ of $r[h]$ satisfies the classical Yang-Baxter equation

$$
\left[r_{1}^{1,2}, r_{1}^{1,3}\right]+\left[r_{1}^{1,2}, r_{1}^{2,3}\right]+\left[r_{1}^{1,3}, r_{1}^{2,3}\right]=0 .
$$

The corresponding deformed right differential map acts on $\mathcal{L} \subset \mathcal{T}$,

$$
\begin{aligned}
\vec{d}[h](L) & =\left(\operatorname{id}_{\mathcal{T}} \otimes \varepsilon_{1}\right)\left(R[h]\left(L^{1}+L^{2}\right) R[h]^{-1}-L \otimes 1_{\mathcal{T}}\right) \\
& =1_{\mathcal{T}} \otimes L+\left[r_{1}, L^{1}+L^{2}\right] h+o\left(h^{2}\right),
\end{aligned}
$$

where we use the expansions $[7]$

$$
R[h]=1_{\mathcal{T} \otimes \mathcal{T}}+r[h]+o\left(h^{2}\right), \quad R[h]^{-1}=1_{\mathcal{T} \otimes \mathcal{T}}-r[h]+o\left(h^{2}\right) .
$$

Similarly

$$
\overleftarrow{d}[h](L)=L \otimes 1_{\mathcal{T}}+\left[r_{1}, L^{1}+L^{2}\right] h+o\left(h^{2}\right) .
$$

In particular the Lie bialgebra cobracket $\delta$ determined by the quasitriangular coboundary structure $r_{1}$ is expressed in terms of the first order coefficients 
of the differential maps by

$$
\delta(L)=\left[r_{1}-\tau_{(2,1)} r_{1}, L^{1}+L^{2}\right]=\left(\vec{d}_{1}-\tau_{(2,1)} \overleftarrow{d}_{1}\right)(L),
$$

thus

$$
\delta=\left(\vec{d}_{1}-\tau_{(2,1)} \stackrel{\leftarrow}{d}_{1}\right) \uparrow_{\mathcal{L}} .
$$

In a subsequent paper we shall show how to quantise more general Lie bialgebras $(\mathcal{L}, \delta)$ by constructing corresponding pairs of differential maps satisfying (21).

Appendix. In response to a referee, we append an explicit description of the antipode $S$. It is given by

$$
\begin{aligned}
& (S \alpha)_{N}=\tau_{N}\left\{\sum_{p=0}^{N-1}(-1)^{N+p} \sum_{N \leq j_{1}<\cdots<j_{p}<N+p}\right. \\
& \left.\left(\mu_{N+1}^{j_{1}, j_{1}+1} \circ \cdots \circ \mu_{N+p}^{j_{p}, j_{p}+1}\right)\left(\alpha_{N+p}\right)\right\},
\end{aligned}
$$

where the reversing permutation map $\tau_{N}$ is the linear map on $\bigotimes^{N} \mathcal{L}$ which reverses the order of components of product tensors, and the map $\mu_{N+k}^{j, j+1}$ : $\otimes^{N+k} \mathcal{L} \rightarrow \bigotimes^{N+k-1} \mathcal{L}$ acts by applying the multiplication map $\mu: \mathcal{L} \otimes \mathcal{L} \rightarrow \mathcal{L}$ between the $j$ th and $(j+1)$ th copies of $\mathcal{L}$ in $\bigotimes^{N+k} \mathcal{L}$. Equivalently $S$ is defined by linear extension of its action on homogeneous product tensors

$$
S\left(L_{1} \otimes \cdots \otimes L_{N}\right)=(-1)^{N}\left(0, \lambda_{1}, \ldots, \lambda_{N}, 0,0, \ldots\right),
$$

where for $1 \leq r \leq N$,

$$
\lambda_{r}=\sum_{1 \leq k_{1}<\cdots<k_{r-1}<N} L_{k_{r-1}+1} \ldots L_{N} \otimes L_{k_{r-2}+1} \ldots L_{k_{r-1}} \otimes \cdots \otimes L_{1} \ldots L_{k_{1}}
$$

in particular $\lambda_{N}=L_{N} \otimes \cdots \otimes L_{1}$, so that when $\mu=0$ we obtain the usual shuffle product antipode.

We denote by $i$ the unit map $\mathbb{F} \ni z \mapsto(z, 0,0, \ldots) \in \mathcal{T}(\mathcal{L})$ and by $M$ the multiplication map $\mathcal{T}(\mathcal{L}) \otimes \mathcal{T}(\mathcal{L}) \rightarrow \mathcal{T}(\mathcal{L})$ given by linear extension of $R \otimes S \mapsto T$ with $T$ given by (1).

TheOREM 6. The map $S$ is an antipode for the bialgebra $(\mathcal{T}(\mathcal{L}), M, i, \Delta, \varepsilon)$.

Proof. We must show [2] that $M \circ\left(S \otimes \mathrm{id}_{\mathcal{T}(\mathcal{L})}\right) \circ \Delta=M \circ\left(\mathrm{id}_{\mathcal{T}(\mathcal{L})} \otimes S\right) \circ \Delta$ $=i \circ \varepsilon$.

Let us prove that $M \circ\left(S \otimes \operatorname{id}_{\mathcal{T}(\mathcal{L})}\right) \circ \Delta=i \circ \varepsilon$. For arbitrary $T=$ $\left(T_{0}, T_{1}, T_{2}, \ldots\right) \in \mathcal{T}(\mathcal{L})$ we have

$$
\begin{aligned}
\left(\left(M \circ\left(S \otimes \operatorname{id}_{\mathcal{T}(\mathcal{L})}\right) \circ \Delta\right) T\right)_{0} & =\left(\left(M \circ\left(S \otimes \operatorname{id}_{\mathcal{T}(\mathcal{L})}\right)\right)(\Delta T)\right)_{0} \\
& =\left(T_{0}, 0,0, \ldots\right)=(i \circ \varepsilon) T .
\end{aligned}
$$


The proof is completed by showing that $\left(M \circ\left(S \otimes \mathrm{id}_{\mathcal{T}(\mathcal{L})}\right) \circ \Delta\right) T=0$ when $(i \circ \varepsilon) T=0$, that is, when $T_{0}=0$. By linearity it is sufficient to consider the case when $T=L_{1} \otimes \cdots \otimes L_{N}$ is a homogeneous product vector. In view of (23),

$$
\begin{aligned}
(M \circ & \left.\left(S \otimes \operatorname{id}_{\mathcal{T}(\mathcal{L})}\right) \circ \Delta\right)\left(L_{1} \otimes \cdots \otimes L_{N}\right) \\
& =\sum_{j=0}^{N}\left(M \circ\left(S \otimes \operatorname{id}_{\mathcal{T}(\mathcal{L})}\right)\right)\left(\left(L_{1} \otimes \cdots \otimes L_{j}\right) \otimes\left(L_{j+1} \otimes \cdots \otimes L_{N}\right)\right) \\
& =\left(L_{1} \otimes \cdots \otimes L_{N}\right)+\sum_{j=1}^{N}(-1)^{j} \sum_{r=1}^{j} \lambda_{r}^{j} \nu_{j},
\end{aligned}
$$

where, for $1 \leq r \leq j$,

$$
\lambda_{r}^{j}=\sum_{1 \leq k_{1}<\cdots<k_{r-1}<j}\left(L_{k_{r-1}+1} \ldots L_{j} \otimes L_{k_{r-2}+1} \ldots L_{k_{r}} \otimes \cdots \otimes L_{1} \ldots L_{k_{1}}\right)
$$

and

$$
\nu_{j}=L_{j+1} \otimes \cdots \otimes L_{N} .
$$

We compute the actions on $\lambda_{r}^{j}$ and $\nu_{j}$ of the left differential map $\overleftarrow{d}$ :

$$
\begin{aligned}
\overleftarrow{d}\left(\lambda_{r}^{j}\right) & =\sum_{1 \leq k_{2}<\cdots<k_{r-1}<j}\left(\sum_{k_{1}=1}^{k_{2}-1} L_{k_{r-1}+1} \ldots L_{j}\right. \\
\overleftarrow{d} \nu_{j} & =L_{j+1} \otimes \nu_{j+1} .
\end{aligned}
$$

Using the Leibniz-Itô formula we find from (24) that

$$
\begin{aligned}
& \sum_{1 \leq k_{2}<\cdots<k_{r-1}<j} \sum_{k_{1}=1}^{k_{2}-1}\left\{L_{k_{r-1}+1} \ldots L_{j} \otimes\left(L_{k_{r-2}+1} \ldots L_{k_{r}} \otimes \cdots \otimes L_{1} \ldots L_{k_{1}}\right) \nu_{j}\right. \\
+ & L_{j+1} \otimes\left(L_{k_{r-1}+1} \ldots L_{j} \otimes L_{k_{r-2}+1} \ldots L_{k_{r}} \otimes \cdots \otimes L_{1} \ldots L_{k_{1}}\right) \nu_{j+1} \\
+ & \left.L_{k_{r-1}+1} \ldots L_{j} L_{j+1} \otimes\left(L_{k_{r-2}+1} \ldots L_{k_{r}} \otimes \cdots \otimes L_{1} \ldots L_{k_{1}}\right) \nu_{j+1}\right\} .
\end{aligned}
$$

When $j=1$ in the sum we obtain the terms

$$
-L_{1} \otimes \nu_{1}-L_{2} \otimes\left(L_{1}\right) \nu_{2}-L_{1} L_{2} \otimes \nu_{2}
$$

of which the first cancels the initial term in (25), leaving

$$
-L_{2} \otimes\left(L_{1}\right) \nu_{2}-L_{1} L_{2} \otimes \nu_{2}
$$

which cancel some of the six terms which occur when $j=2$, which are

$$
\begin{aligned}
L_{1} L_{2} \otimes \nu_{2}+L_{3} \otimes & \left(L_{1} L_{2}\right) \nu_{3}+L_{1} L_{2} L_{3} \otimes\left(L_{1} L_{2}\right) \nu_{3} \\
& +L_{2} \otimes\left(L_{1}\right) \nu_{2}+L_{3} \otimes\left(L_{2} \otimes L_{1}\right) \nu_{3}+L_{2} L_{3} \otimes\left(L_{1}\right) \nu_{3} .
\end{aligned}
$$


After cancellation with (26) the remaining terms

$$
\begin{aligned}
& +L_{3} \otimes\left(L_{1} L_{2}\right) \nu_{3}+L_{1} L_{2} L_{3} \otimes\left(L_{1} L_{2}\right) \nu_{3} \\
& +L_{3} \otimes\left(L_{2} \otimes L_{1}\right) \nu_{3}+L_{2} L_{3} \otimes\left(L_{1}\right) \nu_{3}
\end{aligned}
$$

cancel some of the terms which occur when $j=3$. For general $j$, terms in which $L_{j+1}$ does not occur either alone or multiplied by one or more $L_{k}$ with $k \leq j$ in the left component of the tensor product in $\mathcal{L} \otimes \mathcal{T}(\mathcal{L})$ cancel with terms labelled in the sum by $j-1$, whereas terms in which it does occur cancel terms labelled by $j+1$. When $j=N$ all terms are of the former type and we obtain complete cancellation. We conclude that

$$
\overleftarrow{d}\left(\left(M \circ\left(S \otimes \operatorname{id}_{\mathcal{T}(\mathcal{L})}\right) \circ \Delta\right)\left(L_{1} \otimes \cdots \otimes L_{N}\right)\right)=0,
$$

from which it follows that

$$
\left(M \circ\left(S \otimes \operatorname{id}_{\mathcal{T}(\mathcal{L})}\right) \circ \Delta\right)\left(L_{1} \otimes \cdots \otimes L_{N}\right)=(i \circ \varepsilon)\left(L_{1} \otimes \cdots \otimes L_{N}\right)=0 .
$$

The proof that $M \circ\left(\operatorname{id}_{\mathcal{T}(\mathcal{L})} \otimes S\right) \circ \Delta=i \circ \varepsilon$ is similar but makes use of the right differential map instead of the left.

\section{References}

[1] P. Etingof and D. Kazhdan, Quantization of Lie bialgebras I, Selecta Math. 2 (1996), 1-41; II, ibid. 4 (1998), 213-231; III ibid. 4 (1998), 233-269.

[2] P. Etingof and O. Schiffman, Lectures on Quantum Groups, International Press, 1998.

[3] R. L. Hudson, Calculus in enveloping algebras, J. London Math. Soc. (2) 65 (2001), 361-380.

[4] - Itô versus Woronowicz calculus in the Itô-Hopf algebra, Math. Slovaca 54 (2004), 151-159.

[5] R. L. Hudson and S. Pulmannová, Chaotic expansion of elements of the universal enveloping algebra of a Lie algebra associated with a quantum stochastic calculus, Proc. London Math. Soc. (3) 77 (1998), 462-480.

[6] -, - Double product integrals and Enriquez quantization of Lie bialgebras $I$ : the quasitriangularity relations, J. Math. Phys. 45 (2004), 2090-2105.

[7] —, - Double product integrals and Enriquez quantization of Lie bialgebras II: the quantum Yang Baxter equation, Lett. Math. Phys. 72 (2005), 211-224,

[8] S. L. Woronowicz, Differential calculus on compact matrix pseudogroups, Comm. Math. Phys. 122 (1989), 125-170.

Department of Mathematical Sciences

Loughborough University

Loughborough, Leicestershire

LE11 3TU, Great Britain

E-mail: r.hudson@lboro.ac.uk
Mathematical Institute Slovak Academy of Sciences Stefankova 49

81473 Bratislava, Slovakia E-mail: pulmann@mat.savba.sk 